\title{
Cardiac resynchronization in Poland - comparable procedural routines? Insights from CRT Survey II
}

\author{
Mateusz Tajstra ${ }^{1}$, Damian Łasocha ${ }^{2}$, Elżbieta Gadula-Gacek ${ }^{1}$, Mateusz Ostręga ${ }^{1}$, Lidia Michalak ${ }^{3}$, \\ Dariusz Wojciechowski ${ }^{4}$, Marek Zieliński ${ }^{5}$, Maciej Kempa ${ }^{6}$, Zbigniew Orski ${ }^{7}$, Anna Polewczyk ${ }^{8}$, Jerzy Ozga ${ }^{9}$, \\ Camilla Normand ${ }^{10}$, Kenneth Dickstein ${ }^{10}$, Cecilia Linde ${ }^{11}$, Jarosław Kaźmierczak ${ }^{12}$, Łukasz Szumowski², \\ Mariusz Gąsior ${ }^{1}$, Maciej Sterliński

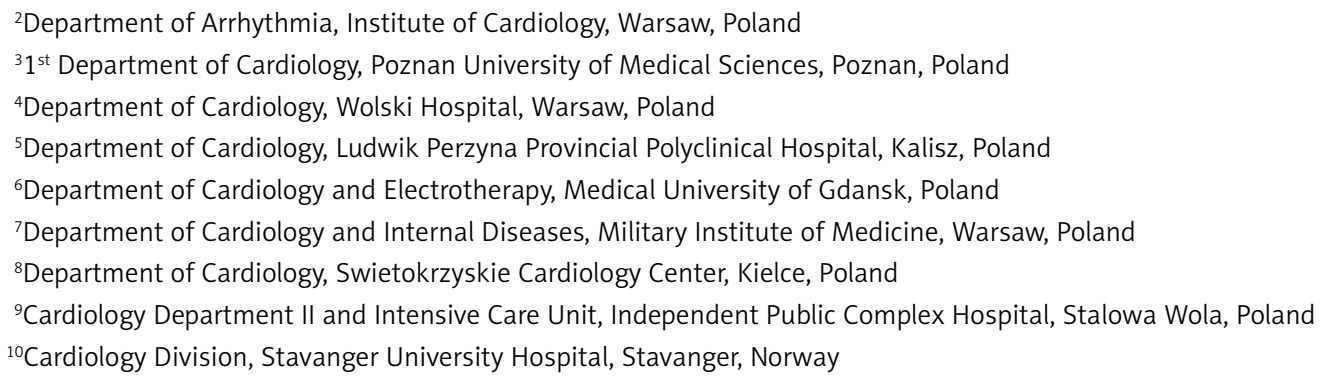

\section{A bstract}

Introduction: CRT Survey II was initiated by the European Heart Rhythm Association and the Heart Failure Association, to explore everyday implantation practice of cardiac resynchronization therapy (CRT) devices in a broad spectrum of hospitals in European Society of Cardiology (ESC) member countries.

Aim: To compare Polish and European procedural practice.

Material and methods: Procedural details of Polish patients collected in 37 Polish centres ( $n=1241$ - Poland group) were compared to the patients enrolled throughout Europe ( $n=9847-$ CRT II Survey group).

Results: There were significant differences in: successful implantation ( $96.1 \%$ vs. $97.4 \%)$, type of device implanted (for CRT-D: $87 \%$ vs. $67.6 \%$ ), implanting physician subspecialty (for electrophysiologist: $69.2 \%$ vs. $79.8 \%$ ), type of location of procedure (for operating room: $19.4 \%$ vs. $8.9 \%$ ), duration of procedure (117.8 \pm 44 vs. $97.5 \pm 46.1 \mathrm{~min}$ ), left ventricle lead type (for multipolar lead: $50 \%$ vs. $57.9 \%)$, coronary sinus venogram with occlusion rate $(41.4 \%$ vs. $47.9 \%)$ and peri-procedural complication rate $(7.5 \%$ vs. $5.3 \%)$ between Poland and CRT II Survey groups, respectively.

Conclusions: This study provides important information describing current differences in Polish procedural routines in relation to ESC member countries. Heterogeneous CRT implantation practices across European countries still exist. However, it may be related to different clinical profile of patients qualified for CRT implantation in Poland as well as organization of care.

Key words: cardiac resynchronisation therapy, chronic heart failure, survey.

Su m m a ry

Details related to the implantation procedure of the cardiac resynchronization therapy (CRT) may be one of the most important factors that could potentially have a significant impact on response to resynchronization therapy. The clinical implications from this investigation can be summarized as follows: first, the percentage of successful attempts of CRT implantation in the entire cohort was high (about 97\%); second, CRT implantation practices may vary across European countries; third, in Poland CRT-P is generally less frequently implanted; finally, the periprocedural complication rate was higher in Poland in comparison to the rest of Europe, although this fact could be related to clinical profile and more frequently used combined antithrombotic therapy.

Corresponding author:

Dr Mateusz Tajstra, $3^{\text {rd }}$ Department of Cardiology, Faculty of Medical Sciences in Zabrze, Medical University of Silesia, 2 Szpitalna St, 41-800 Zabrze, Poland, phone: +48 3237336 19, e-mail: mateusztajstra@wp.pl

Received: 27.03.2019, accepted: 1.07.2019. 


\section{Introduction}

Benefits of cardiac resynchronization therapy (CRT) have been clinically proven in randomized clinical trials (RCTs) [1-7]. Therefore, in current practice guidelines CRT is an unambiguously and strongly advocated treatment option in patients with heart failure (HF) $[8,9]$. However, in clinical practice patients are often very much older and with more comorbidities than in RCTs.

Details related to the implantation procedure of the CRT may be one of the most important factors that could potentially have a significant impact on response to resynchronization therapy. However, the majority of the currently available data regarding CRT implantation routines and peri-procedural complications have been obtained from RCTs performed mostly in relatively high volume centres by experienced implanters. Therefore, widespread recommendations regarding CRT implementation, including clinical status, ECG findings and optimal implantation techniques, are based explicitly on data from RCTs. However, the first CRT survey demonstrated that a large number of CRT implantation procedures were not performed in line with the guidelines [10] and some details of procedural techniques also varied between participating centres [11].

Moreover, evidence from surveys and registry-based analyses that encompass everyday clinical practice, meaning all eligible, consecutive and high risk patients, may add to RCTs in creation of evidence-based medicine [12].

\section{Aim}

Bearing in mind all the facts mentioned above and having at our disposal a large group of patients with CRT implantation details coming from the CRT II Survey - a joint project between the European Heart Rhythm Association (EHRA) and the Heart Failure Association (HFA) - we aimed to compare Polish and European procedural practice associated with CRT implantations in patients enrolled in the CRT II Survey.

\section{Material and methods}

\section{Survey infrastructure}

The survey was designed as a joint initiative between EHRA and the HFA. The design and rationale of CRT Survey II, along with the detailed contents of the eCRFs, have been published previously [13]. Briefly, the 47 European Society of Cardiology (ESC) member states detailed in the 2014 EHRA White Book were invited to participate [14]. Finally, 42 ESC member countries participated in the survey. The survey gathered data on patients' characteristics, investigations, indications for CRT, implant procedures and short-term outcome including adverse events. Each implanting centre was asked to provide information regarding facility type, size, and operator speciality. Information on operator experience was not collected.

\section{Survey population}

Any patient in the 42 participating countries was eligible for inclusion if they were selected and implanted with either a CRT with pacemaker function (CRT-P) or a CRT with an incorporated defibrillator (CRT-D). This included both successful and unsuccessful implantations as well as both de-novo CRT devices and upgrades from a previous permanent pacemaker (PPM) or implanted cardiac defibrillator (ICD). Generator replacements or revisions of previously implanted CRT devices were excluded as the survey was designed to cover only de novo CRT implantations or upgrades from a previous PM or ICD. Specific data regarding an implantation procedure included inter alia: location of procedure, duration and fluoroscopy time, left ventricular (LV) lead type placement and its position X-ray evaluation, percentage of coronary venogram performance, and periprocedural complications. Information on lead insertion (venesection or puncture) was not collected.

\section{Study population}

A total of 288 centres from 42 European countries enrolled data of a total of 11088 patients implanted with a CRT device with or without a cardioverter-defibrillator between October 2015 and December 2016. In this cohort of patients 1241 (11.2\%) patients recruited in 37 Polish centres - university, regional, and private (Poland group) - are presented in comparison to the total 9847 (88.8\%) patients enrolled throughout Europe (CRT II Survey group).

\section{Statistical analysis}

Absolute numbers and percentages were shown for categorical variables to describe the patient population, and means (with standard deviations) or medians (with interquartile range) were used for continuous variables. Categorical variables were compared between subgroups by the $\chi^{2}$ test and continuous variables (numerical values) by the Mann-Whitney-Wilcoxon test. A significance level of $p<0.05$ was assumed for the statistical tests. All statistical analyses were performed using SAS statistical software (version 9.3, Cary, NC, USA).

\section{Results}

The principal clinical implications from this investigation can be summarized as follows: first, the percentage of successful attempts of CRT implantation in the entire cohort was high (about 97\%); second, CRT implantation practices may vary across European countries; third, in Poland CRT-P is generally less frequently implanted; finally, the periprocedural complication rate was higher in Poland in comparison to the rest of Europe.

Poland was the greatest participant in the CRT Survey with more than $12 \%$ of the total patient material. Polish patients were younger with more ischemic heart disease, 
more often received an CRT-D and more often were upgraded from a previous PM or ICD.

We found important differences in Polish procedural routines in relation to the rest of participating European countries, indicating that heterogeneous CRT implantation practices across European countries may still exist.

The baseline clinical characteristics of the study groups are presented in Table I. On the whole, despite younger age, patients treated in Poland had a more severe clinical profile with, among other things, higher rates of: ischemic cardiomyopathy $(58.5 \%$ vs. $42.7 \%$, $p<0.001)$, previous myocardial infarction (48.4\% vs.
$34.7 \%, p<0.001$ ), previous coronary revascularization (51.2\% vs. $37.3 \%, p<0.001$ ), atrial fibrillation (43.8\% vs. $40.5 \%, p=0.03)$ and lower mean left ventricle ejection fraction $(26.4 \%$ vs. $28.7 \%, p<0.001)$.

General outcome data are presented in Table II. There were 1250 implants attempts in Poland and 9960 in the CRT II Survey group, and 1191 (96.1\%) successful in the Poland group vs. 9606 (97.4\%) in the CRT II Survey group. Procedural details of the study groups are presented in Table III and Figures 1-3. Among others, significant differences were observed in: previous device implantation (30.7\% vs. $27.5 \%, p=0.02$ ), type of device implanted (for

Table I. Baseline clinical characteristics of study groups

\begin{tabular}{|c|c|c|c|}
\hline \multirow[t]{2}{*}{ Variable } & Poland & CRT II Survey & \multirow[t]{2}{*}{$P$-value } \\
\hline & $n=1241(11.2 \%)$ & $n=9847(88.8 \%)$ & \\
\hline Age, mean \pm SD [years] & $67.7 \pm 9.7$ & $68.6 \pm 10.9$ & $<0.001$ \\
\hline Males & $81.3 \%$ & $75 \%$ & $<0.001$ \\
\hline Ischemic cardiomyopathy & $58.5 \%$ & $42.7 \%$ & $<0.001$ \\
\hline Hypertension & $68.4 \%$ & $63.3 \%$ & $<0.001$ \\
\hline Diabetes & $37.2 \%$ & $30.7 \%$ & $<0.001$ \\
\hline Prior myocardial infarction & $48.4 \%$ & $34.7 \%$ & $<0.001$ \\
\hline Prior coronary revascularization & $51.2 \%$ & $37.3 \%$ & $<0.001$ \\
\hline Valvular heart disease & $32.2 \%$ & $26.5 \%$ & $<0.001$ \\
\hline Chronic kidney disease (class $\leq 3$ ) & $36 \%$ & $30.5 \%$ & $<0.001$ \\
\hline Mean LV-EF, mean \pm SD (\%) & $26.4 \pm 8$ & $28.7 \pm 8.1$ & $<0.001$ \\
\hline Atrial fibrillation & $43.8 \%$ & $40.5 \%$ & 0.03 \\
\hline
\end{tabular}

CRT-D - cardiac resynchronization therapy-defibrillator, LV-EF - left ventricle ejection fraction, SD - standard deviation.

Table II. Baseline clinical characteristics of study groups

\begin{tabular}{|c|c|c|c|}
\hline \multirow[t]{2}{*}{ Variable } & Poland & CRT II Survey & \multirow[t]{2}{*}{$P$-value } \\
\hline & $n=1241(11.2 \%)$ & $n=9847(88.8 \%)$ & \\
\hline Age, mean \pm SD [years] & $67.7 \pm 9.7$ & $68.6 \pm 10.9$ & $<0.001$ \\
\hline Males & $81.3 \%$ & $75 \%$ & $<0.001$ \\
\hline Ischemic cardiomyopathy & $58.5 \%$ & $42.7 \%$ & $<0.001$ \\
\hline Hypertension & $68.4 \%$ & $63.3 \%$ & $<0.001$ \\
\hline Diabetes & $37.2 \%$ & $30.7 \%$ & $<0.001$ \\
\hline Prior myocardial infarction & $48.4 \%$ & $34.7 \%$ & $<0.001$ \\
\hline Prior coronary revascularization & $51.2 \%$ & $37.3 \%$ & $<0.001$ \\
\hline Valvular heart disease & $32.2 \%$ & $26.5 \%$ & $<0.001$ \\
\hline Chronic kidney disease (class $\leq 3$ ) & $36 \%$ & $30.5 \%$ & $<0.001$ \\
\hline Mean LV-EF, mean \pm SD (\%) & $26.4 \pm 8$ & $28.7 \pm 8.1$ & $<0.001$ \\
\hline Atrial fibrillation & $43.8 \%$ & $40.5 \%$ & 0.03 \\
\hline
\end{tabular}

CRT-D - cardiac resynchronization therapy-defibrillator, LV-EF - left ventricle ejection fraction, SD - standard deviation. 
Table III. Procedural details of the study groups

\begin{tabular}{|c|c|c|c|}
\hline \multirow[t]{2}{*}{ Variable } & Poland & CRT II Survey & \multirow[t]{2}{*}{$P$-value } \\
\hline & $n=1241(11.2 \%)$ & $n=9847(88.8 \%)$ & \\
\hline Admission to implantation, mean \pm SD [days] & $3.4 \pm 5.2$ & $3.9 \pm 9.8$ & $<0.001$ \\
\hline Previous device implantation & $30.7 \%$ & $27.5 \%$ & 0.02 \\
\hline Successful implantation attempt & $96.1 \%$ & $97.4 \%$ & 0.008 \\
\hline Unsuccessful attempt & $3.9 \%$ & $2.6 \%$ & \\
\hline LV lead placement unsuccessful* & $87.2 \%$ & $88.8 \%$ & \\
\hline Type of device: & & & $<0.001$ \\
\hline CRT-D implantation & $87 \%$ & $67.6 \%$ & \\
\hline CRT-P implantation & $13 \%$ & $32.4 \%$ & \\
\hline Operator: & & & $<0.001$ \\
\hline Electrophysiologist & $69.2 \%$ & $79.8 \%$ & \\
\hline HF physician & $2.6 \%$ & $5.3 \%$ & \\
\hline Invasive cardiologist & $24.3 \%$ & $10.9 \%$ & \\
\hline Surgeon & $0 \%$ & $4.8 \%$ & \\
\hline Other & $3.9 \%$ & $1.0 \%$ & \\
\hline Location of procedure: & & & $<0.001$ \\
\hline Cathlab & $23.7 \%$ & $25.5 \%$ & \\
\hline Dedicated electrophysiological lab & $16.6 \%$ & $32.4 \%$ & \\
\hline Device implantation lab & $40.2 \%$ & $32.6 \%$ & \\
\hline Operating room & $19.4 \%$ & $8.9 \%$ & \\
\hline Other & $0.1 \%$ & $0.6 \%$ & \\
\hline Duration of procedure, mean \pm SD [min] & $117.8 \pm 44.1$ & $97.5 \pm 46.1$ & $<0.001$ \\
\hline Fluoroscopy time, mean \pm SD [min] & $20.4 \pm 15.6$ & $17.4 \pm 17.3$ & $<0.001$ \\
\hline Prophylactic antibiotics & $99.2 \%$ & $98.6 \%$ & 0.06 \\
\hline Test shock & $8.4 \%$ & $4.3 \%$ & $<0.001$ \\
\hline Which lead was implanted first: & & & NS \\
\hline RV & $82.9 \%$ & $83.6 \%$ & \\
\hline LV & $17.1 \%$ & $16.4 \%$ & \\
\hline LV lead type: & & & $<0.001$ \\
\hline Unipolar & $1.5 \%$ & $0.6 \%$ & \\
\hline Bipolar & $48.5 \%$ & $41.4 \%$ & \\
\hline Multipolar & $50.0 \%$ & $57.9 \%$ & \\
\hline Coronary sinus venogram performed & $92.6 \%$ & $91.4 \%$ & NS \\
\hline Venogram performed with occlusion & $41.4 \%$ & $47.9 \%$ & $<0.001$ \\
\hline Dilatation of coronary vein performed & $2.4 \%$ & $2.4 \%$ & NS \\
\hline Phrenic nerve stimulation tested & $86.5 \%$ & $90.9 \%$ & $<0.001$ \\
\hline LV lead position optimization & $20.1 \%$ & $35.6 \%$ & $<0.001$ \\
\hline
\end{tabular}

*Percentage of non-successful attempts. CRT-D - cardiac resynchronization therapy-defibrillator, CRT-P - cardiac resynchronization therapy-pacemaker, LV - left ventricle, $R V$-right ventricle, NS - not significant, SD - standard deviation. 
Poland

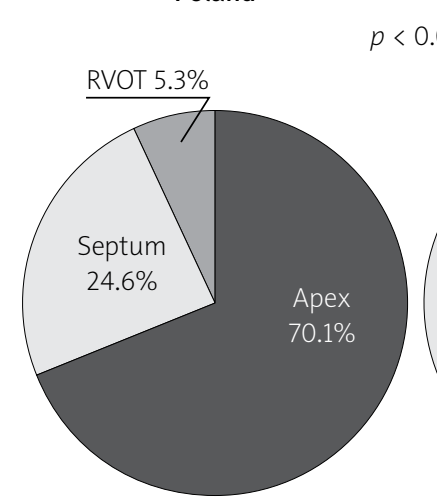

CRT II Survey 0.001

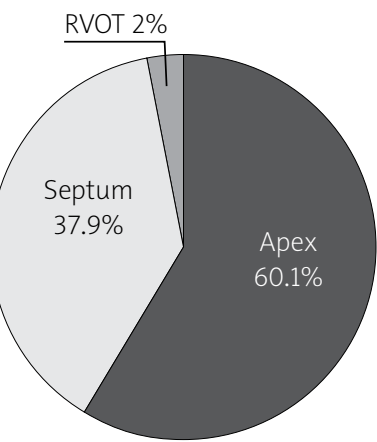

Figure 1. Right ventricle lead placement

CRT-D: $87 \%$ vs. $67.6 \%, p<0.001$ ), type of operator (for electrophysiologist: $69.2 \%$ vs. $79.8 \%, p<0.001$ ), type of location of procedure (for operating room: $19.4 \%$ vs. $8.9 \%, p<0.001)$, procedure duration (117.8 \pm 44 vs. 97.5 $\pm 46.1 \mathrm{~min}, p<0.001$ ), left ventricle lead type (for multipolar lead: $50 \%$ vs. $57.9 \%, p<0.001$ ), and coronary sinus venogram with occlusion rate $(41.4 \%$ vs. $47.9 \%$, $p<0.001$ ) between Poland and CRT II Survey groups.

Additionally, the periprocedural complication rate was higher in the Polish group than in the CRT Survey group ( $7.5 \%$ vs. $5.3 \%, p=0.001)$ (Table IV). In particular pneumothorax and coronary sinus dissection were more common in Poland. This might be associated either with lead insertion technique and operator experience or with higher percentage of Polish patients with dual antiplatelet $(13.9 \%$ vs. $8.7 \%, p<0.001)$ and triple anticoagulation therapy $(2.9 \%$ vs. $1.9 \%, p=0.02)$ (Table V).

\section{Discussion}

Within the last decade cardiac resynchronisation therapy (CRT) has been proven to be a highly effective
Poland

CRT II Survey

$$
p<0.001
$$
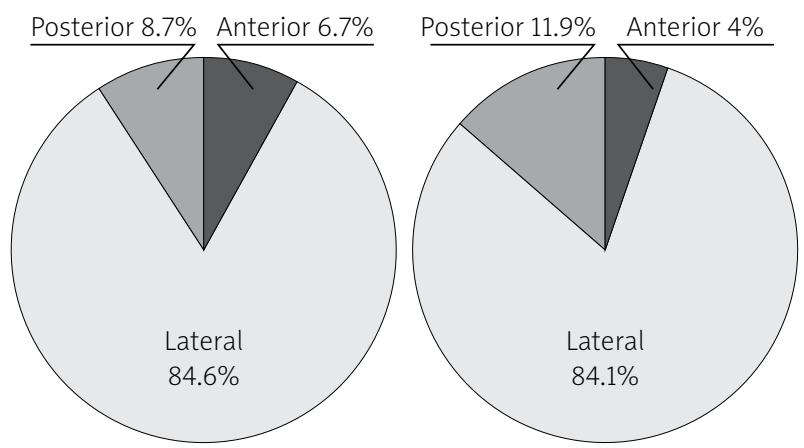

Figure 2. Left ventricle lead position. Left anterior oblique site evaluation

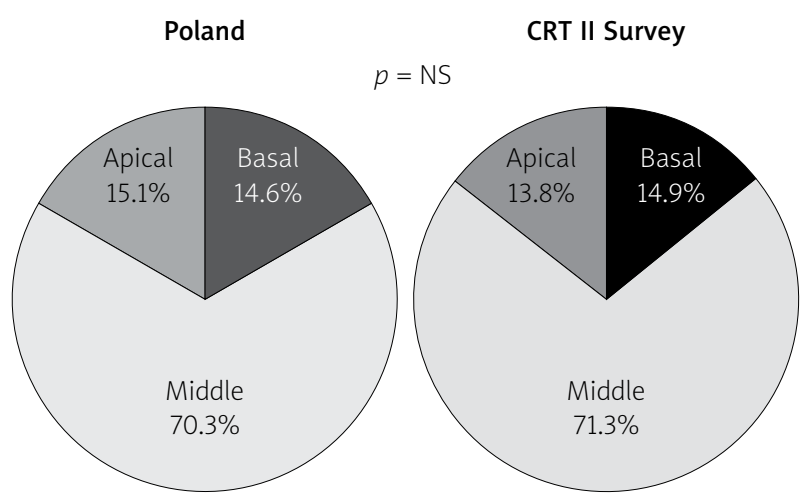

Figure 3. Left ventricle lead position. Right anterior oblique site evaluation

therapy to reduce morbidity and mortality in chronic HF patients [1-7]. Therefore, current practice guidelines strongly support CRT implantation in optimally pharmacologically treated patients. It is estimated that about 400 patients per million per year might be suitable for

Table IV. Periprocedural complications

\begin{tabular}{|c|c|c|c|}
\hline \multirow[t]{2}{*}{ Variable } & Poland & CRT II Survey & $P$-value \\
\hline & $n=1241(11.2 \%)$ & $n=9847(88.8 \%)$ & \\
\hline Periprocedural complication: & $7.5 \%$ & $5.3 \%$ & 0.001 \\
\hline Death* & $1.1 \%$ & $1.3 \%$ & NS \\
\hline Bleeding*: & $18.1 \%$ & $17.4 \%$ & NS \\
\hline Requiring intervention* & $41.2 \%$ & $31.9 \%$ & \\
\hline Pocket haematoma* & $88.2 \%$ & $76.9 \%$ & \\
\hline Pneumothorax & $17 \%$ & $18.5 \%$ & NS \\
\hline Haemothorax* & $2.1 \%$ & $1.3 \%$ & NS \\
\hline Coronary sinus dissection* & $38.3 \%$ & $38.6 \%$ & NS \\
\hline Pericardial tamponade* & $6.4 \%$ & $3.8 \%$ & NS \\
\hline Other* & $22.3 \%$ & $27.6 \%$ & NS \\
\hline
\end{tabular}

*Percentage of periprocedural complications. CRT - cardiac resynchronization therapy, NS - not significant. 
Table V. Drug therapy at discharge

\begin{tabular}{|c|c|c|c|}
\hline \multirow[t]{2}{*}{ Variable } & Poland & CRT II Survey & $P$-value \\
\hline & $n=1241(11.2 \%)$ & $n=9847(88.8 \%)$ & \\
\hline$\beta$-blocker & $96.4 \%$ & $88 \%$ & $<0.001$ \\
\hline ACEI/ARB & $90.1 \%$ & $85.9 \%$ & $<0.001$ \\
\hline MRA & $78.7 \%$ & $61.2 \%$ & $<0.001$ \\
\hline Loop diuretic & $88.9 \%$ & $80 \%$ & $<0.001$ \\
\hline Ivabradine & $7.9 \%$ & $5.3 \%$ & $<0.001$ \\
\hline Digoxin & $11.3 \%$ & $10.3 \%$ & 0.29 \\
\hline Amiodarone & $13.7 \%$ & $17.8 \%$ & $<0.001$ \\
\hline Anti-platelet agent & $53.3 \%$ & $42.5 \%$ & $<0.001$ \\
\hline DAPT & $13.9 \%$ & $8.7 \%$ & $<0.001$ \\
\hline Oral anticoagulation and P2Y12 inhibitor & $5.1 \%$ & $4.0 \%$ & 0.06 \\
\hline Triple therapy & $2.9 \%$ & $1.9 \%$ & 0.02 \\
\hline
\end{tabular}

ACE-I - angiotensin enzyme converting inhibitor, ARB - angiotensin receptor blocker, DAPT - dual antiplatelet therapy, MRA - mineralocorticoid receptor antagonist.

CRT, or up to 400000 patients per year in ESC countries [8]. Accordingly, we are deeply convinced that the knowledge about everyday clinical practice, including procedural aspects and periprocedural complications related to CRT implantation procedures, will allow us to responsibly improve current standards. Such data, encompassing details about consecutive, real-life, non-selected procedures, can be delivered by surveys and registries.

Cardiac Resynchronization Therapy Survey II provided precious information regarding CRT implantation routines in nearly three hundred centres from 42 European countries. One of the main goals of the survey was to make representative benchmarking both nationally and internationally accessible. Thus, in this substudy we compared Polish and European CRT routines. Polish centres participating have included the largest number of patients. We focused on the technical issues related to CRT implantation procedures - one of the most important factors in the potential response to resynchronization therapy.

Safe and effective CRT device implantation requires the appropriate environment, trained personnel, equipment and optimally located left ventricle (LV) lead. These aspects may affect the frequency, especially of early but also long-term complications.

Considering the above-mentioned "appropriate environment and trained personnel", as far as we are concerned, the link between operator type or location of procedure and type of CRT used has not been studied previously. We found that invasive cardiologists were more likely to implant CRT among eligible patients in Poland in comparison to other participating countries. The underlying reasons for this association and its potential impact on the procedural and especially long-term out- come remain unknown. In our opinion, further research to compare CRT implantations according to clinician training, type of the operator specialty, operator experience and place of the procedure may provide insights into strategies to further optimize CRT use.

Although CRT Survey II did not provide information regarding complications due to a venous access and operator experience, it was previously proved that the blind puncture approach of the subclavian or axillary vein is associated with a higher risk of pneumothorax [15]. There are no direct studies concerning operator experience and complication rate in patients with CRT. However, the risk of CRT implantation infection was significantly higher in patients who had their CRT implanted by an inexperienced operator [16].

Regarding "equipment", this study shows that the multipolar LV lead was a frequent option in the entire cohort. LV lead selection may not only result in more frequent challenges posed during implantation of the CRT system, but also allows to facilitate the optimal outcome in terms of obtaining an adequate capture threshold and avoidance of phrenic nerve stimulation. Multipolar leads offer various pacing configurations to minimize phrenic nerve capture and may permit reprogramming to alternate stimulation vectors to ameliorate this problem without re-intervention [17-19]. The use of a multipolar lead may also be a promising option for patients with ischemic $\mathrm{HF}$ and the presence of a scar around the LV lead placement is potentially an important factor of decrease of CRT response. However, the application of multisite pacing using a multipolar lead to increase CRT effectiveness has been investigated with conflicting results [20,21].

Another issue regards differences between de novo CRT implantations and upgrades from previous devices. 
The latest studies demonstrated that implantation procedures in upgraded patients were equally successful and complications similar to de novo implantations [22].

Following "optimally located LV lead" mainly (above $80 \%$ ) and similarly in both groups lateral LV lead position as assessed in the left anterior oblique site was recorded. However, in the Poland group anterior LV lead position was more frequent. Right anterior oblique evaluation, primarily middle placement (about 70\%), was observed equally in compared groups. In spite of the fact that there is increasing evidence proving that selection of specific LV sites for pacing may improve CRT outcomes, no data are available from any large RCT and therefore no clear recommendations have been introduced; however, LV lead placement in anterior veins should be avoided. Sub-analysis reports of two major randomized clinical trials support lateral and deny apical placement. The REVERSE study has suggested that lateral position of the LV lead is associated with better outcome in terms of reverse remodelling and results collected from the MADIT-CRT trial have revealed that apical placement of the LV lead is associated with a less favourable outcome [23, 24].

Although there is increasing evidence of the benefit of optimizing the LV positions if the anatomy allows for choices, attempts to optimize LV lead position during implantation overall were not intense and were made less frequently in the Polish group ( $20.1 \%$ vs. $35.6 \%)$. The preferred LV lead placement should be over the region of the latest mechanical activation. This approach is supported by the results of the TARGET (Targeted Left Ventricular Lead Placement to Guide Cardiac Resynchronization Therapy) trial, which showed that positioning of the LV lead at the latest activated region resulted in a better echocardiographic and clinical response and less HF hospitalization [25]. Likewise, the position of the LV lead in the region of the latest electrical activation often applied using QLV has been linked to a greater response to CRT [26]. A possible explanation for the reluctance to perform systematic optimisation of the LV lead position is that such an approach might lack a routine to use QLV or other electrical dyssynchrony criteria during the procedure or imaging techniques considered as more demanding for the implanting cardiologists and its advantages are yet to be proved in large randomized clinical trials.

Discussing the results in Polish and the whole CRT Survey groups, there are some major differences (i.e. percentage of complications, optimal LV lead placement and fluoroscopy time). In our opinion, Poland should focus on training so that more experienced operators can perform safe and effective procedures.

\section{Limitations}

Several limitations to the current study should be stressed. Surveys have their own limitations which need to be acknowledged and considered during interpreta- tion of findings. Differences in practice between countries can skew the results [27]. Centre participation was voluntary and we estimate that about $11 \%$ of patients implanted with CRT in participating countries were enrolled in the survey. Although consecutive inclusion was strongly encouraged, we cannot confirm that all patients were included consecutively. The accuracy of the data has not been audited and there may be a potential selection bias. However, the investigators conducted a 'front-end' data check and post database lock quality control analyses designed to prevent incorrect data being analysed.

\section{Conclusions}

This study provides important information describing current Polish differences in procedural routines in relation to the rest of participating European countries. Heterogeneous CRT implantation practices across European countries may still exist. However, it may be related to a different clinical profile of patients qualified for CRT implantation in Poland.

\section{Acknowledgments}

Mateusz Tajstra and Damian Łasocha equally contributed to the study.

\section{Conflict of interest}

The authors declare no conflict of interest.

\section{References}

1. Cazeau S, Leclercq C, Lavergne T, et al. Effects of multisite biventricular pacing in patients with heart failure and intraventricular conduction delay. N Engl J Med 2001; 344: 873-80.

2. Abraham WT, Fisher WG, Smith AL, et al. Cardiac resynchronization in chronic heart failure. N Engl J Med 2002; 346: 1845-53.

3. Bristow MR, Saxon LA, Boehmer J, et al. Cardiac-resynchronization therapy with or without an implantable defibrillator in advanced chronic heart failure. N Engl J Med 2004; 350: 2140-50.

4. Cleland JG, Daubert JC, Erdmann E, et al. The effect of cardiac resynchronization on morbidity and mortality in heart failure. N Engl J Med 2005; 352: 1539-49.

5. Linde C, Abraham WT, Gold MR, et al. Randomized trial of cardiac resynchronization in mildly symptomatic heart failure patients and in asymptomatic patients with left ventricular dysfunction and previous heart failure symptoms. J Am Coll Cardiol 2008; 52: 1834-43.

6. Moss AJ, Hall WJ, Cannom DS, et al. Cardiac-resynchronization therapy for the prevention of heart-failure events. N Engl J Med 2009; 361: 1329-38.

7. Tang AS, Wells GA, Talajic M, et al. Cardiac-resynchronization therapy for mild-to-moderate heart failure. N Engl J Med 2010; 363: 2385-95.

8. Raatikainen MJP, Arnar DO, Merkely B, et al. A Decade of Information on the Use of Cardiac Implantable Electronic Devices and Interventional Electrophysiological Procedures in the European Society of Cardiology Countries: 2017 Report from the European Heart Rhythm Association. Europace 20171; 19: ii1-90. 
9. Ponikowski P, Voors AA, Anker SD, et al.; Authors/Task Force Members. 2016 ESC Guidelines formthe diagnosis and treatment of acute and chronic heart failure:The TaskForce forthe diagnosis and treatment of acute and chronic heart failure of the European Society of Cardiology (ESC)Developed with the special contribution of the Heart Failure Association (HFA) of the ESC. Eur Heart J 2016; 37: 2129-200.

10. Dickstein K, Bogale N, Priori S, et al.; Scientific Committee; National Coordinators. The European cardiac resynchronization therapy survey. Eur Heart J 2009; 30: 2450-60.

11. Bogale N, Priori S, Gitt A, et al.; Scientific Committee, National coordinators, and investigators. The European cardiac resynchronization therapy survey: patient selection and implantation practice vary according to centre volume. Europace 2011; 13 : 1445-53.

12. Gitt AK, Bueno H, Danchin N, et al. The role of cardiac registries in evidence-based medicine. Eur Heart J 2010; 31: 525-9.

13. Dickstein K, Normand C, Anker SD, et al. European cardiac resynchronization therapy survey II: rationale and design. Europace 2015; 17: 137-41.

14. Raatikainen MJ, Arnar DO, Zeppenfeld K, et al. Statistics on the use of cardiac electronic devices and electrophysiological procedures in the European Society of Cardiology countries: 2014 report from the European Heart Rhythm Association. Europace 2015; 17: 1-75.

15. Van Rees JB, de Bie MK, Thijssen J, et al. Implantation-related complications of implantable cardioverter-defibrillators and cardiac resynchronization therapy devices: a systematic review of randomized clinical trials. J Am Coll Cardiol 2011; 58: 995-1000.

16. Polyzos KA, Konstantelias AA, Falagas ME. Risk factors for cardiac implantable electronic device infection: a systematic review and meta-analysis. Europace 2015; 17: 767-77.

17. Gurevitz O, Nof E, Carasso S, et al. Programmable multiple pacing configurations help to overcome high left ventricular pacing thresholds and avoid phrenic nerve stimulation. Pacing Clin Electrophysiol 2005; 28: 1255-9.

18. Forleo GB, Della Rocca DG, Papavasileiou LP, et al. Left ventricular pacing with a new quadripolar transvenous lead for CRT: early results of a prospective comparison with conventional implant outcomes. Heart Rhythm 2011; 8: 31-7.

19. Shetty AK, Duckett SG, Bostock J, et al. Use of a quadripolar left ventricular lead to achieve successful implantation in patients with previous failed attempts at cardiac resynchronization therapy. Europace 2011; 13: 992-6.

20. Van Gelder BM, Bracke FA. Acute hemodynamic effects of singleand dual-site left ventricular pacing employing a dual cathodal coronary sinus lead. Pacing Clin Electrophysiol 2015; 38: 558-64.

21. Sterliński M, Sokal A, Lenarczyk R, et al. In heart failure patients with left bundle branch block single lead multispot left ventricular pacing does not improve acute hemodynamic response to conventional biventricular pacing. a multicenter prospective, interventional, non-randomized study. PLoS One 2016; 11: e0154024.

22. Linde CM, Normand C, Bogale N, et al. Upgrades from a previous device compared to de novo cardiac resynchronization therapy in the European Society of Cardiology CRT Survey II. Eur J Heart Fail 2018; 20: 1457-68.

23. Thebault C, Donal E, Meunier C, et al. Sites of left and right ventricular lead implantation and response to cardiac resynchronization therapy observations from the REVERSE trial. Eur Heart J 2012; 33: 2662-71
24. Singh JP, Klein HU, Huang DT, et al. Left ventricular lead position and clinical outcome in the multicenter automatic defibrillator implantation trial-cardiac resynchronization therapy (MADITCRT) trial. Circulation 2011; 123: 1159-66.

25. Khan FZ, Virdee MS, Palmer CR, et al. Targeted left ventricular lead placement to guide cardiac resynchronization therapy: the TARGET study: a randomized, controlled trial. J Am Coll Cardiol 2012; 59: 1509-18.

26. Chatterjee NA, Gold MR, Waggoner AD, et al. Longer left ventricular electric delay reduces mitral regurgitation after cardiac resynchronization therapy: mechanistic insights from the SMART-AV Study (Smart Delay Determined AV Optimization: A Comparison to Other AV Delay Methods Used in Cardiac Resynchronization Therapy). Circ Arrhythm Electrophysiol 2016; 9: e004346.

27. Swedberg K, Cleland J, Cowie MR, et al. Successful treatment of heart failure with devices requires collaboration. Eur J Heart Fail 2008; 10: 1229-35. 